\section{Practical applications of the shell model}

Shell-Model Applications in Nuclear Spectroscopy. By P. J. Brussaard and P. W. M. Glaudemans. Pp.452 (North Holland: Amsterdam, New York and Oxford, 1977.) Dfl.160; \$65.50.

THE shell model is the most basic and comprehensive of all nuclear models, and in the past three decades it has been devcloped to a high degree of sophistication and extensively used to analyse nuclear spectroscopic data. Nucleons interact strongly, so it is at first sight rather surprising that they can be assigned independent orbits inside the nucleus, but this comes about because of the Pauli Exclusion Principle that forbids many collisions that would otherwise occur and also the rapid falloff of the nucleon-nucleon interaction with distance. Early calculations did not give the observed sequence of magic numbers of nucleons corresponding to closed shells, but this was remedied with the addition of the spinorbit potential in 1949. Thereafter, the shell model has proved astonishingly succesful in accounting for a wide range of nuclear reaction and structure data.

The book by Brussaard and Glaudemans is a welcome addition to the already considerable literature on the shell model. It is a comprehensive textbook at the level of the first-year graduate student, with detailed chapters on the more important aspects of the model. The formalism is derived in detail, so that it is useful for reference as well as for self-study.

After an introductory chapter on the foundation of the shell model, the formalism for one- and two-particle systems is described, and perturbation theory is applied to calculate binding and excitation energies. Shell model calculations require the evaluation of matrix elements of interaction operators between many-particle states, taking account of configuration mixing and using angular momentum coupling and coefficients of fractional parentage. This is described in detail and applied to the cases of interacting particles in one and two orbits.

The dimension of the configuration space in shell model calculations increases very rapidly with the number of active particles. It is therefore usual to truncate the space and use an effective interaction the parameters of which are adjusted to fit selected data. A particularly successful interaction is the surface delta interaction, and this is applied to several nuclei.

Shell-model wavefunctions are used to calculate the cross-sections of oneand two-nucleon transfer reactions, and this is onc of the principal ways of determining spectroscopic factors. Electromagnetic transition operators and rates are also calculated with shell model wavefunctions, as well as electric and magnetic multipole moments and beta decay rates. All these subjects are described, with many practical applications.

Additional chapters summarise the second quantisation formalism, realistic and effective operators, the applications of Wick's theorem, the Lanczos method, Coulomb energies, spurious states and the particle-vibrator model. There are many appendices on coupling coefficients, the Wigner-Eckart theorem, reduced matrix elements, and

\section{Physics in perspective}

A Perspective of Physics. Edited by Rudolph Peierls. Pp. 243. (Gordon and Breach: London, New York and Paris, 1977.) $£ 20$.

$\Upsilon_{\mathrm{HE}}$ rate of progress of significant research in physics remains very high. There is indeed no sign as yet of it falling off, despite the financial strains in the western world during the past few years. Under these circumstances it is essential to have continually available reviews and comments at different levels so that the subject does not become too over-specialised. The so-called Comments Journals dealing with nuclear and particle physics, solid-state physics, astrophysics, atomic and molecular physics, and plasma physics and controlled fusion, fulfill a very useful role in this respect. This particular volume goes further in presenting a selection of comments from all these areas. so that from them the reader may obtain some idca of the growing points in the subject as a whole.

For a volume of this kind to be a success it is essential that the selection be made by some distinguished physicist who does see the subject as a whole. No-one better than Sir Rudolf Peierls could be imagined for this task. Furthermore the value of the book is greatly increased by the masterly introduction by Sir Rudolf which attempts with great success to put the whole into perspective in a scant 26 pages. It is remarkable how wellbalanced is this Introduction. covering as it does such different kinds of research ranging from very fundamental studies in particle physics and in astrophysics through solid-state and atomic physics, and involving a rich variety of tables of 3-j and 6-j symbols, fractional parentage coefficients and the matrix elements for some simple potentials.

This book will certainly be very useful to all who have to learn about the shell model and make practical calculations. Some references to the original work are given, but the value of the book would have been increased by a more comprehensive list. The section on Coulomb energies is too bricf and contains no mention of the Nolan-Schiffer anomaly. The book is excellently printed and the price is correspondingly high.

\section{P. E. Hodgson}

P. E. Hodgson is Lecturer in Nuclear Physics at the University of Oxford, UK.

phenomena depending on known basic laws, to a highly technical subject such as controlled fusion.

There is great excitement today from the new, unexpected discoverics of new particles and new quantum numbers which can hardly be said to have simplified the basic description of Nature. On the other hand very promising developments towards unifying the theories of the weak, clectromagnetic and strong interactions have been made. The weakest of all the interactions. the gravitational. is the one which dominates the exotic aspects of astrophysics in which white dwarfs and neutron stars are commonplace and black holes are on the brink of observational confirmation. In these areas of 'big' science the demands on technology are very severe, so that the most advanced technologies today are to be found in these fundamental research activities. Nevertheless there is a great amount of highly applicable work being done in the smaller sciences, again depending very much on new techniques. After a long period of uncertainty it now looks very probable that power from controlled fusion is a practical possibility, though still a distant one, and high morale exists among those working in this area.

Apart from the Introduction the book includes eight selcctions from Comments on Nuclear and Particle Physics, five each from Solid-State Physics, Astrophysics and Atomic and Molecular Physics and four from Plasma Physics and Controlled Fusion. The selection is a good one and should prove absorbing to a physicist anxious to know something of what is going on around him beyond his own direct involvement.

Harrie Massey

Sir Harrie Massey is Emeritus Professor of Plipsics in the Liniversity of I.ondon, UK. 